\title{
Relationship between Two Job Stress Models and Coronary Risk Factors among Japanese Part-Time Female Employees of a Retail Company
}

\author{
Yuka Kobayashi ${ }^{1}$, Toshio Hirose ${ }^{2}$, Yumiko TAdA ${ }^{2}$, Akizumi Tsutsumi ${ }^{1}$ and \\ Norito KAWAKAMI ${ }^{1}$ \\ ${ }^{1}$ Department of Hygiene and Preventive Medicine, Okayama University Graduate School of Medicine and \\ Dentistry and ${ }^{2}$ Sendai Nishikicho Clinic and Center of Occupational Medicine, Japan
}

\begin{abstract}
Relationship between Two Job Stress Models and Coronary Risk Factors among Japanese Part-Time Female Employees of a Retail Company: Yuka KовaYAshi, et al. Department of Hygiene and Preventive Medicine, Okayama University Graduate School of Medicine and Dentistry-The objective of this study was to explore the associations between two major job stress models (job strain and effort-reward imbalance) and coronary heart disease (CHD) risk factors (blood pressure; total, high- (HDL) and low-density lipoprotein (LDL) cholesterol; and triglycerides) in Japanese part-time female employees of a retail company. The study population was either $35 \mathrm{yr}$ old or between 40 and 63 yr old. Data collection was carried out in 2002; a total of 1,401 subjects participated in a medical examination and completed a questionnaire. After adjusting for other covariates (age, relative weight, tobacco use, alcohol consumption, lack of exercise, education, marital status, history of child bearing, medical treatment for disease, and occupation), a significant association was found between the effort-reward imbalance, a "highcost and low-gain" condition at work, and a high prevalence of low HDL cholesterol (Odds ratio $=4.4$ ). A weak but unexpected association was found between job strain and low prevalence of low HDL cholesterol. In explanatory analysis with individual components of the two models, associations were evident between high extrinsic effort and high prevalence of low HDL cholesterol and low prevalence of high triglyceride, high job control and low prevalence of high systolic blood pressure, and high job demands and low prevalence of high systolic and diastolic blood pressure. In this
\end{abstract}

Received Sep 8, 2004; Accepted Jan 24, 2005

Correspondence to: Y. KOBAYASHI, Department of Hygiene and Preventive Medicine, Okayama University Graduate School of Medicine and Dentistry, 2-5-1 Shikata-cho, Okayama 700-8558, Japan (e-mail: yukakoba@md.okayama-u.ac.jp) cross-sectional study of Japanese part-time working women, a significant association was found between effort-reward imbalance and unfavorable HDL cholesterol profiles. The findings did not support the hypothesis that job strain is associated with CHD risk factors.

(J Occup Health 2005; 47: 201-210)

Key words: Job strain, Effort-reward imbalance, Coronary risk factors, Part-time workers, Women, Japan

In the past two decades, a cumulative body of results has been reported showing an association between job strain defined in Karasek's job strain model ${ }^{1)}$ and coronary heart disease (CHD) ${ }^{2-4)}$ and its risk factors ${ }^{5-17)}$. A high level of job strain, that is, a combination of high level of job demands and low level of job control, has been found to be associated with physiological CHD risk factors, such as high blood pressure in men ${ }^{5-10)}$ and women ${ }^{11)}$, high serum total cholesterol in men ${ }^{9-12)}$ and women ${ }^{9)}$, low highdensity lipoprotein (HDL) cholesterol in men and women $^{11)}$, and high triglycerides in men ${ }^{8)}$. In addition, social support at work appears to reduce the effects of job strain on CHD risk factors, such as high blood pressure in women ${ }^{18)}$ and high serum total cholesterol in men $^{9)}$. However, previous studies have reported no clear association between job strain and high blood pressure in men $^{11,14,15,19,20)}$ and women ${ }^{6,7,9,15,17,20)}$, high serum total cholesterol in men ${ }^{5,8,13,14,20)}$ and women ${ }^{12,13,17,20)}$, low HDL cholesterol in men ${ }^{8,11-13,15,20)}$ and women ${ }^{12,13,15,17,20)}$, and high triglycerides in $\operatorname{men}^{15,20)}$ and women ${ }^{15,17,20)}$.

Another more recently developed job stress model is the effort-reward imbalance model ${ }^{21)}$. This model supposes that the source of stress in the workplace results from imbalance between individual recognition of extrinsic effort (e.g., high workload) and the extrinsic 
reward (e.g., money, esteem, and occupational status control), focusing on the negative trade-off between experienced costs and gains at work. However, this model has been studied much less than the job strain model, which shows that effort-reward imbalance also predicts $\mathrm{CHD}^{22-24)}$. A significant association between effortreward imbalance and high blood pressure, high serum total cholesterol, and high total / HDL cholesterol has been observed in men ${ }^{25}$.

Information on women is sparse and inconsistent when compared to the data from studies of $\operatorname{men}^{6,9,11-13,15,17,20)}$ with regard to the job strain model ${ }^{6,9,11-13,15,17,20)}$, effortreward imbalance model ${ }^{25)}$, and a combination of both ${ }^{23)}$. These differences might be attributable to difficulties in finding associations owing to the protective function of female reproductive hormones and contamination of data from domestic work and job stress. Another important factor related to job stress is whether an individual is employed full- or part-time. Recently, the number of part-time working women has been increasing in Japan ${ }^{26)}$; it has grown by approximately 1.4 times in the last decade. The results of studies in western countries indicate that part-time workers have lower levels of perceived job stress than full-time workers ${ }^{27}$. The low levels of job stress among part-time employees might be attributable to limited exposure to job stressors; however, a Japanese study showed lower levels of decision latitude in female temporary employees than permanent employees ${ }^{28)}$. Furthermore, part-time employees experience more job insecurity and poorer prospects for promotion than fulltimers. Although such differences in job stress are generally attributed to working conditions, it appears that the association between job stress and CHD risk factors in part-time employees has never been examined.

The questionnaires used for the job strain and effortreward imbalance models are similar (i.e., job demands and extrinsic effort); however, the job strain model focuses more on task-oriented characteristics, while the effort-reward imbalance model deals with the broader occupational environment. Furthermore, the questionnaires show that the job strain scale makes use of objective job characteristics ${ }^{29}$, while the effort-reward imbalance questionnaire measures the emotional aspects induced by stressful situations (see the Methods section). Thus, associations with biological CHD risk factors, if any, might be different between the two stress measures. Furthermore, application of the two stress models might provide more comprehensive features of the association between psychosocial job stress and coronary risks.

The objective of this study was to explore the associations between psychosocial work environments and CHD risk factors in a population of Japanese parttime female employees. Japanese retail companies hire many part-time females, and we used a large database of retailers. The two stress models and biological CHD risk factors were measured simultaneously.

\section{Subjects and Methods}

\section{Subjects}

The study population was recruited from a retail business in Miyagi Prefecture, Japan. A total of 5,635 employees were engaged in processing, sales, and the delivery of dairy goods, and about 80 percent of them were part-time employees. The target population included 3,510 female employees who worked four hours daily, 20 hours weekly, five days a week. They were selected from all part-time females $(n=4,104)$, and the majority worked four-hour shifts.

A cross-sectional survey was carried out between July and December 2002 using a mailed questionnaire on demographic variables and the psychosocial work environment. Each employee was asked to complete the questionnaire. They also underwent an annual medical examination, which included a physical examination to determine blood pressure and blood tests. Blood samples were collected from participants aged $35 \mathrm{yr}$ and from those aged $40 \mathrm{yr}$ or above $(\mathrm{n}=2,798)$, according to the Industrial Safety and Health Law and related regulations. One thousand four hundred one participants completed both the questionnaire and medical examination, including the blood test (participation rate: $50 \%$ ).

The study design and procedures were reviewed and approved by the Human Ethics Committee for Epidemiological Research at the Okayama University Graduate School of Medicine and Dentistry, Japan.

\section{Procedures \\ CHD risk factors}

The biological risk factors for CHD were measured as a part of the medical examination. The examination included measurements of blood pressure and serum lipids from blood samples, and took place during working hours (09:30 to 16:00).

The subject was seated, and then blood pressure was measured on the right arm once or twice by trained nurses. Measurements were obtained with a sphygmomanometer. The point at which the Korotkoff sounds disappeared (Swan's point 5) was recorded as the diastolic blood pressure. High systolic blood pressure was defined as $140 \mathrm{mmHg}$ or greater and high diastolic blood pressure was defined as $90 \mathrm{mmHg}$ or greater, according to the guidelines of The Japanese Society of Hypertension ${ }^{30)}$.

Serum total cholesterol, HDL cholesterol, low-density lipoprotein (LDL) cholesterol, and triglycerides were measured from the blood samples using high performance liquid chromatography. High serum total cholesterol was defined as $220 \mathrm{mg} / \mathrm{d} l$ or greater; low HDL cholesterol as under $40 \mathrm{mg} / \mathrm{d} l$; high LDL cholesterol as $140 \mathrm{mg} / \mathrm{d} l$ or greater; and high triglycerides as $150 \mathrm{mg} / \mathrm{d} l$ or greater, according to the guidelines of the Japan Atherosclerosis 
Society $^{31)}$.

\section{Psychosocial job stressors}

Psychosocial job stressors were measured according to two major job stress models, the job strain ${ }^{1)}$ or demandcontrol-support model ${ }^{4)}$ and the effort-reward imbalance model $^{21)}$. The job strain model was measured using the Japanese version of the Job Content Questionnaire (JCQ), which contains 22 Likert-scaled items ${ }^{32,33)}$. Job strain score reflects a relatively objective work environment ${ }^{29}$. A scale of 1 to 4 was used to measure job strain. There were 5 items measuring "job demands" such as "working very fast"; nine items composed of two subscales for "job control": "skill discretion" with 6 items such as "opportunity to develop abilities", and "decision authority" with 3 items such as "allowed to make decisions"; four items for "supervisor support" such as "the supervisor pays attention to me"; and 4 items on "coworker support" such as "coworkers take an interest in me". The questions were designed to seek information about the existence of stressors in the workplace. A 4point scale ranging from strongly disagree to strongly agree was developed, and the scores ranged from 12 to 48 for job demands, 24 to 96 for job control, 4 to 16 for supervisor support, and 4 to 16 for coworker support. The reliability coefficients of each subscale were job demands: 0.65 , job control: 0.72 , supervisor support: 0.90 , and coworker support: 0.77 . The quotient of job demands to job control was used as an indicator of "job strain" according to previous studies ${ }^{19)}$, and ranged from 0.1 to 2.0. The subjects were grouped into one of three strata for each psychosocial job stressor (low, medium or high) based on tertiles defined according to the distribution of scores: $15-31,32-35$, and 36 or greater, respectively, for job demands; 24-58, 59-66, and 67 or greater, respectively, for job control; 4-9, 10-11, and 12 or greater, respectively, for supervisor support; $4-10,11$, and 12 or greater, respectively, for coworker support; and $0.28-0.51$, $0.51-0.61$, and 0.61 or greater, respectively, for the job strain index.

The effort-reward imbalance model was measured using the Japanese version of the effort-reward imbalance questionnaire, which contains 17 Likert-scaled items ${ }^{21,34)}$. Two categories were used to measure these items. One was "extrinsic effort", which had 6 items such as "I am under constant time pressure due to my heavy work load", the other was "extrinsic reward", which had 11 items composed of three subscales. The subscales were as follows: "job promotion and salary" with 4 items such as "My job promotion prospects are poor"; "esteem" with 5 items such as "I receive the respect I deserve from my supervisors"; and "job instability" with 2 items such as "My job security is poor". The extrinsic effort and extrinsic reward categories were designed to seek information from respondents about the existence of stressful environmental conditions. If they agree, they are then asked to indicate the level of distress on a 4point scale ranging from very distressed to not at all distressed. The total score used to measure these elements ranged from 6 to 30 for extrinsic effort and from 11 to 55 for extrinsic reward. The reliability coefficients of each subscale were 0.80 and 0.84 for extrinsic effort and extrinsic reward, respectively. The effort-reward imbalance index was calculated using the following formula, which ranged from 0.2 to 5 :

extrinsic effort $\times 11 /((66 \text {-extrinsic reward }) \times 0.5454)^{35)}$

An effort-reward imbalance index was used to indicate "high-cost and low-gain", which reflects a subjective work environment. The subjects were grouped into one of three strata for each psychosocial job stressor (low, medium or high) based on tertiles defined according to the distribution of scores: $6-10,11-14$, and 15 or greater, respectively, for extrinsic effort; 11-14, 15-20, and 21 or greater, respectively, for extrinsic reward; and 0.20 $0.37,0.37-0.56$, and 0.56 or greater, respectively, for the effort-reward imbalance index.

\section{Covariates}

Subjects were divided into three age groups according to age, 35 and 40-44, 45-49, and 50-63 yr old, and three occupational categories were used, laborers, clerks, or others. Other covariates included relative weight, healthrelated behaviors, and demographic variables. Height and weight were measured by trained nurses during the medical examination. The degree of relative weight was calculated as the relative excess in weight to a standard weight based on height $\left(22 \times\right.$ height $\left.(\mathrm{m})^{2}\right)$. Relative weight was divided into three categories: less than $-10 \%$, between -10 and $10 \%$, and greater than $10 \%$.

Health-related behaviors included tobacco use, alcohol consumption, and lack of exercise, and were self-reported. Tobacco use was determined according to whether individuals classified themselves as a current smoker, exsmoker, or non-smoker; they were further divided into current or non-smokers. Alcohol consumption was determined according to how individuals classified their alcohol use: that is, daily, occasional, or rare; they were further divided into drinkers (those who answered "daily" or "occasional") and non-drinkers (those who answered "rare"). Frequency of exercise was determined by individual answers as follows: "more than once weekly", "sometimes", and "rarely"; subjects were then divided into one of two groups, "once weekly" or "less than once weekly"; the latter was defined as a lack of exercise.

Educational level was determined by years of school completed, and subjects were divided into two groups, $12 \mathrm{yr}$ or less of schooling or more than $12 \mathrm{yr}$. Two categories were used for marital status, currently married or not married. Subjects also indicated whether or not 
they had children and whether they were being treated for CHD-related diseases, such as hypertension, hyperlipidemia, angina pectoris, myocardial infarction, and diabetes.

\section{Statistical analysis}

First, associations of psychosocial job stressors and tobacco use, alcohol consumption, lack of exercise, and relative weight were examined by the $\chi^{2}$ test. Next, the associations between psychosocial job stressors and CHD risk factors were examined by multiple logistic regressions. Four psychosocial job stressors (job strain, supervisor support, coworker support, or effort-reward imbalance) were entered into the model to predict each CHD risk factor (the prevalence of high systolic/diastolic blood pressure, high total cholesterol, low HDL cholesterol, high LDL cholesterol, and high triglycerides), while controlling all covariates (age, tobacco use, alcohol consumption, lack of exercise, education, marital status, child bearing history, and medical treatment for disease) and occupation. As explanatory analyses, similar multiple logistic regression of each CHD risk factor were conducted with regards to job demands, job control, extrinsic effort, extrinsic reward, supervisor support, and coworker support, while controlling all covariates. All associations were inferred with an $\alpha$ level of 0.05 . These were performed using SPSS computer program, version 11 (Chicago, IL, U.S.A.).

\section{Results}

The characteristics of the subjects are presented in Table 1. Compared with the excluded subjects, the number of clerical and married employees was higher ( 5 vs. $8 \%, \chi^{2}=13.4 ; p=0.001$, and 96 vs. $98 \%, \chi^{2}=5.8 ; p=$ 0.016 , respectively) among those analyzed. There were no differences between the analyzed and excluded subjects with regards to the other studied variables.

The associations of job stressors and tobacco use,

Table 1. Characteristics of the study sample

\begin{tabular}{|c|c|c|}
\hline Variables & $\mathrm{n}$ & $(\%)$ \\
\hline \multicolumn{3}{|c|}{ Sociodemographic data and health-related behaviors } \\
\hline \multicolumn{3}{|l|}{ Age (years) } \\
\hline 35 or $40-44$ & 337 & $(24.1)$ \\
\hline $45-49$ & 465 & $(33.2)$ \\
\hline $50-63$ & 599 & $(42.8)$ \\
\hline \multicolumn{3}{|c|}{ Relative weight (relative excess in weight, see text) } \\
\hline$<-10 \%$ & 209 & $(14.9)$ \\
\hline$-10-10 \%$ & 776 & $(55.4)$ \\
\hline $10 \%<$ & 416 & $(29.7)$ \\
\hline \multicolumn{3}{|l|}{ Tabaccouse } \\
\hline Current smoker & 182 & $(13.0)$ \\
\hline Never or ex-smoker & 1219 & $(87.0)$ \\
\hline \multicolumn{3}{|l|}{ Alcohol consumption } \\
\hline Daily or sometimes & 634 & $(45.3)$ \\
\hline Rarely & 767 & $(54.7)$ \\
\hline \multicolumn{3}{|l|}{ Exercise } \\
\hline Once weekly & 346 & $(24.7)$ \\
\hline Less than once weekly & 1055 & $(75.3)$ \\
\hline \multicolumn{3}{|l|}{ Education } \\
\hline 12 years or less & 1015 & $(72.4)$ \\
\hline More than 12 years & 386 & $(27.6)$ \\
\hline \multicolumn{3}{|l|}{ Marital status } \\
\hline Married & 1369 & $(97.7)$ \\
\hline Not married & 32 & $(2.3)$ \\
\hline \multicolumn{3}{|l|}{ Childbearing } \\
\hline Yes & 768 & $(54.8)$ \\
\hline No & 633 & $(45.2)$ \\
\hline \multicolumn{3}{|l|}{ Being treated for diseases $\left.{ }^{a}\right)$} \\
\hline Yes & 135 & $(9.6)$ \\
\hline No & 1266 & $(90.4)$ \\
\hline
\end{tabular}


(continued)

Table 1. Characteristics of the study sample

\begin{tabular}{|c|c|c|}
\hline Variables & $\mathrm{n}$ & $(\%)$ \\
\hline \multicolumn{3}{|l|}{ Occupation } \\
\hline Production line workers & 1241 & $(88.6)$ \\
\hline Clerks & 116 & $(8.3)$ \\
\hline Others & 44 & $(3.1)$ \\
\hline \multicolumn{3}{|c|}{ Psychosocial job stressors (scores in the parentheses) } \\
\hline \multicolumn{3}{|l|}{ Job strain (Job demands/Job control ratio) } \\
\hline Low $(.28-.51)$ & 375 & $(26.8)$ \\
\hline Medium (.51-.61) & 489 & $(34.9)$ \\
\hline High (.61-1.62) & 537 & $(38.3)$ \\
\hline \multicolumn{3}{|l|}{ Supervisor support } \\
\hline High (12-16) & 671 & $(47.9)$ \\
\hline Medium (10-11) & 305 & $(21.8)$ \\
\hline Low (4-9) & 425 & $(30.3)$ \\
\hline \multicolumn{3}{|l|}{ Coworker support } \\
\hline High (12-16) & 726 & $(51.8)$ \\
\hline Medium (11) & 371 & $(26.5)$ \\
\hline Low (4-10) & 304 & $(21.7)$ \\
\hline \multicolumn{3}{|l|}{ Effort-reward imbalance } \\
\hline Low $(.20-.37)$ & 479 & $(34.2)$ \\
\hline Medium (.37-.56) & 460 & $(32.8)$ \\
\hline High (.56-2.08) & 462 & $(33.0)$ \\
\hline \multicolumn{3}{|l|}{ CHD risk factors } \\
\hline High systolic blood pressure ( $\geq 140 \mathrm{mmHg}$ ) & 149 & $(10.6)$ \\
\hline High diastolic blood pressure ( $\geq 90 \mathrm{mmHg}$ ) & 136 & $(9.7)$ \\
\hline High total cholesterol $(\geq 220 \mathrm{mg} / \mathrm{d} l)$ & 452 & $(32.3)$ \\
\hline Low HDL cholesterol $(<40 \mathrm{mg} / \mathrm{d} l)$ & 40 & $(2.9)$ \\
\hline High LDL cholesterol ( $\geq 140 \mathrm{mg} / \mathrm{d} l$ ) & 434 & $(31.0)$ \\
\hline High triglycerides $(\geq 150 \mathrm{mg} / \mathrm{d} l)$ & 175 & $(12.5)$ \\
\hline
\end{tabular}

a)“diseases" comprises hypertension, hyperlipidemia, angina pectoris, myocardial infarction, and diabetes.

alcohol consumption, lack of exercise, and relative weight are shown in Table 2. In our subjects, there were no statistically significant associations, although those who reported low coworker support tended to exercise less frequently $\left(\chi^{2}=5.6, p<0.10\right)$.

When four psychosocial job stressors and all other covariates were simultaneously entered into the multiple logistic regression model (Table 3), part-time women exposed to the highest effort-reward imbalance were four times as likely to be associated with high prevalence of low HDL cholesterol compared with those with the lowest effort-reward imbalance. On the other hand, although the association was weaker, the high job strain group had a significantly lower odds ratio (OR) of high prevalence of low HDL cholesterol. Associations between social support and CHD risk factors were apparent, but not statistically significant. Age and relative weight were significantly associated with all CHD risk factors except for HDL cholesterol; tobacco use was inversely associated with high prevalence of high total cholesterol; alcohol consumption was inversely associated with high prevalence of high total and LDL cholesterol; and lack of exercise was significantly associated with high prevalence of high systolic blood pressure. Additional controlling for occupation did not change the results.

Explanatory logistic regression analyses using job demands, job control, extrinsic effort, extrinsic reward, supervisor support, and coworker support as independent variables showed that high extrinsic effort was associated with high prevalence of low HDL cholesterol (OR $=2.92$, 95\% confidence interval $(\mathrm{CI})=1.04-8.19$ for the medium group; $\mathrm{OR}=4.23,95 \% \mathrm{CI}=1.34-13.38$ for the high group), and low prevalence of high triglycerides $(\mathrm{OR}=0.83,95 \%$ $\mathrm{CI}=0.55-1.26$ for the medium group; $\mathrm{OR}=0.46,95 \%$ $\mathrm{CI}=0.27-0.78$ for the high group). As expected, high job control was associated with low prevalence of high systolic blood pressure $(\mathrm{OR}=0.75,95 \% \mathrm{CI}=0.48-1.15$ for the medium group; $\mathrm{OR}=0.43,95 \% \mathrm{CI}=0.26-0.71$ for the 
Table 2. Association of psychosocial job stressors with health-related behaviors (tobacco use, alcohol consumption, and lack of exercise) and relative weight status among part-time working women ${ }^{\text {a) }}$

\begin{tabular}{|c|c|c|c|c|c|c|c|c|c|c|c|c|}
\hline \multirow{3}{*}{$\begin{array}{l}\text { Job stressor } \\
\text { (Range of scores) }\end{array}$} & \multirow{2}{*}{\multicolumn{2}{|c|}{ Current smoker }} & \multirow{2}{*}{\multicolumn{2}{|c|}{$\begin{array}{c}\text { Drinker } \\
\text { (daily or } \\
\text { sometimes) }\end{array}$}} & \multirow{2}{*}{\multicolumn{2}{|c|}{$\begin{array}{l}\text { Lack of exercise } \\
\text { (Less than } \\
\text { once weekly) }\end{array}$}} & \multicolumn{6}{|c|}{ Relative weight ${ }^{\mathrm{b}}$ ) } \\
\hline & & & & & & & \multicolumn{2}{|c|}{$<-10 \%$} & \multicolumn{2}{|c|}{$-10-10 \%$} & \multicolumn{2}{|c|}{$10 \%<$} \\
\hline & $\mathrm{n}$ & $(\%)$ & $\mathrm{n}$ & $(\%)$ & $\mathrm{n}$ & $(\%)$ & $\mathrm{n}$ & $(\%)$ & $\mathrm{n}$ & $(\%)$ & $\mathrm{n}$ & $(\%)$ \\
\hline \multicolumn{13}{|c|}{ Job strain (Job demands/Job control ratio) } \\
\hline Low $(.28-.51)$ & 46 & $(12.3)$ & 173 & $(46.1)$ & 292 & $(77.9)$ & 51 & $(13.6)$ & 205 & $(54.7)$ & 119 & $(31.7)$ \\
\hline Medium (.51-.61) & 69 & $(14.1)$ & 218 & $(44.6)$ & 356 & $(72.8)$ & 75 & $(15.3)$ & 283 & $(57.9)$ & 131 & $(26.8)$ \\
\hline High $(.61-1.62)$ & 67 & $(12.5)$ & 243 & $(45.3)$ & 407 & $(75.8)$ & 83 & $(15.5)$ & 288 & $(53.6)$ & 166 & $(30.9)$ \\
\hline \multicolumn{13}{|l|}{ Supervisor support } \\
\hline High (12-16) & 90 & $(13.4)$ & 295 & $(44.0)$ & 513 & $(76.5)$ & 110 & $(16.4)$ & 366 & $(54.5)$ & 195 & $(29.1)$ \\
\hline Medium (10-11) & 37 & $(12.1)$ & 147 & $(48.2)$ & 232 & $(76.1)$ & 45 & $(14.8)$ & 164 & $(53.8)$ & 96 & $(31.5)$ \\
\hline Low (4-9) & 55 & $(12.9)$ & 192 & $(45.2)$ & 310 & $(72.9)$ & 54 & $(12.7)$ & 246 & $(57.9)$ & 125 & $(29.4)$ \\
\hline \multicolumn{13}{|l|}{ Coworker support } \\
\hline High (12-16) & 92 & $(12.7)$ & 321 & $(44.2)$ & 549 & $(75.6)$ & 118 & $(16.3)$ & 393 & $(54.1)$ & 215 & $(29.6)$ \\
\hline Medium (11) & 47 & $(12.7)$ & 164 & $(44.2)$ & 265 & $(71.4)$ & 55 & $(14.8)$ & 211 & $(56.9)$ & 105 & $(28.3)$ \\
\hline Low (4-10) & 43 & $(14.1)$ & 149 & $(49.0)$ & 241 & $(79.3)$ & 36 & $(11.8)$ & 172 & $(56.6)$ & 96 & $(31.6)$ \\
\hline \multicolumn{13}{|c|}{ Effort-reward imbalance } \\
\hline Low $(.20-.37)$ & 60 & $(12.5)$ & 213 & $(44.5)$ & 360 & $(75.2)$ & 81 & $(16.9)$ & 256 & $(53.4)$ & 142 & $(29.6)$ \\
\hline Medium (.37-.56) & 62 & $(13.5)$ & 211 & $(45.9)$ & 359 & $(78.0)$ & 67 & $(14.6)$ & 269 & $(58.5)$ & 124 & $(27.0)$ \\
\hline High (.56-2.08) & 60 & $(13.0)$ & 210 & $(45.5)$ & 336 & $(72.7)$ & 61 & $(13.2)$ & 251 & $(54.3)$ & 150 & $(32.5)$ \\
\hline
\end{tabular}

a) No significant association was observed between job stressors and health-related behaviors (tobacco use, alcohol consumption, or lack of exercise) or relative weight status in crude or age-adjusted analysis ( $\chi^{2}$ test or multiple logistic regression, respectively, $p>0.05$ ).

b) Relative excess (\%) in weight. See text.

high group), but unexpectedly high job demands were associated with low prevalence of high systolic blood pressure $(\mathrm{OR}=0.60,95 \% \mathrm{CI}=0.37-0.96$ for the medium group; $\mathrm{OR}=0.71,95 \% \mathrm{CI}=0.44-1.16$ for the high group) and low prevalence of high diastolic blood pressure $(\mathrm{OR}=0.45,95 \% \mathrm{CI}=0.27-0.76$ for the medium group; $\mathrm{OR}=0.86,95 \% \mathrm{CI}=0.53-1.41$ for the high group). Although not statistically significant, job demands showed a tendency to be associated with low prevalence of low HDL cholesterol $(\mathrm{OR}=0.92,95 \% \mathrm{CI}=0.36-2.36$ for the medium group; $\mathrm{OR}=0.80,95 \% \mathrm{CI}=0.31-2.06$ for the high group). Low coworker support was significantly associated with high prevalence of low HDL cholesterol $(\mathrm{OR}=2.21,95 \% \mathrm{CI}=1.03-4.76$ for the medium group; $\mathrm{OR}=1.35,95 \% \mathrm{CI}=0.55-3.30$ for the low group).

\section{Discussion}

Multivariate logistic regression analyses revealed a significant association between effort-reward imbalance and high prevalence of low HDL cholesterol in female part-time employees of a retail business, after adjusting for relevant confounding factors (age, relative weight, tobacco use, alcohol consumption, lack of exercise, education, marital status, child bearing, medical treatment of disease, and occupation). On the other hand, high job strain was associated with low prevalence of low HDL cholesterol. Explanatory analyses showed expected findings with regards to job control and support components, whereas demand components, extrinsic effort, and particularly, job demands had inverse associations with CHD risk factors. The associations between covariates and CHD risk factors were mostly as expected.

Effort-reward imbalance was associated with a large proportion of individuals with low HDL cholesterol. On the other hand, as in previous studies with inconsistent results ranging from a significantly adverse association ${ }^{11)}$ to almost no association ${ }^{12,13,15,17,20)}$ in women, this study showed high HDL cholesterol in the part-time workers exposed to job strain. These results might have been caused by differences in the aspects measured by both models. Emotional reactions to unpleasant situations might be an important variable in biochemical correlation studies of cholesterol ${ }^{36)}$, and lipid elevation appears to be more affected by perceived stress than objective stress ${ }^{37)}$. The job strain model might be related more to objective measures of stressors than other questionnaires ${ }^{29)}$; the subjects are questioned about their job characteristics, not about their feelings concerning stress. In contrast, the effort-reward questionnaire approaches more 


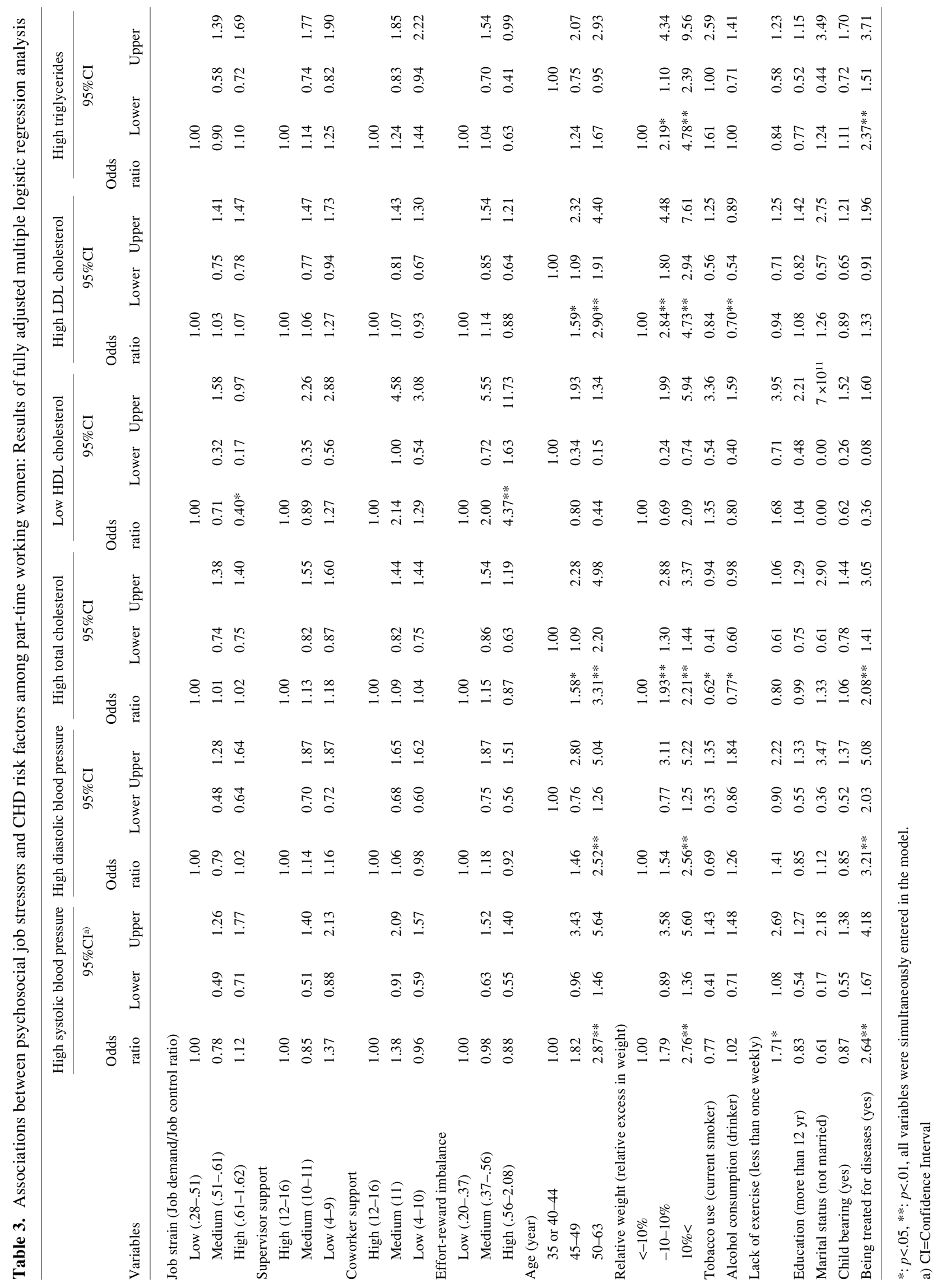


cognitive levels of perceived job stress, and in this questionnaire the subjects are asked how they feel distressed.

The two stress models might provide an additional explanation for the results on lipid profiles. Previous investigations of occupational instability and job insecurity have frequently demonstrated increased total cholesterol levels ${ }^{38,39)}$ and, to a lesser extent, high blood pressure levels ${ }^{40)}$. Thus, perceived threat of job loss or poor job prospects could be a sensitive predictor of lipid deterioration, which might have been replicated in the effort-reward imbalance analysis. However, prevalence of low HDL cholesterol was very low; although relevant confounders were adjusted for in this study, the subjects with low HDL cholesterol may be a special group characterized by factors other than effort-reward imbalance, such as poor health practices or lifestyles. Further studies are necessary to explore the association between effort-reward imbalance and poor HDL cholesterol profiles.

The explanatory analysis revealed that the unexpected findings with regards to job strain appeared to be primarily attributable to the job demands component. The majority of recent evidence on job strain and CHD has not been able to confirm the full model, that is, the combination with high demands and low control ${ }^{41)}$. Most recent studies have predicted an association between low control and CHD risk, whereas high demands were associated with decreased CHD risk. A demanding situation might not necessarily lead to adverse consequences in the current working world. This proposition might apply to Siegrist's effort component ${ }^{21)}$ to some extent (i.e., the unexpected observed association with triglycerides), and it requires more study. As expected, however, part-time women with high job control had low prevalence of high systolic blood pressure. Extrinsic effort was also associated with unfavorable HDL profiles, but the combination of high extrinsic effort and low extrinsic reward showed a clearer association, as postulated by the model ${ }^{21)}$.

Many tests showed no statistically significant associations, not only in our main hypothesis between job stressors and biological CHD risk factors but also in the associations between job stressors and health-related behaviors, which are possible mediators through which job stressors lead to biological CHD risk factors. These findings might be related to the specific characteristics of the sample studied. Part-time workers in retail businesses, such as the one studied here, consist of a central work force. While career development is limited, employees do not face a severe threat of job loss. In addition, exposure to adverse conditions is limited because of the limited working hours. Thus, it should be taken into account that the sample did not necessarily represent part-time employees who hypothetically experience harsh working conditions in terms of low occupational rewards.

The following limitations of the present study need to be discussed. First, because of the cross-sectional design of the study, a causal relationship was not determined by these results. Another shortcoming due to the study design is related to the employees' response to psychosocial job characteristics. Most employees have an annual physical checkup and were aware of their health status. Those who were aware that their health status was poor might have let their health situation be affected by external work conditions. More definite evidence would be obtained from a longitudinal study ${ }^{24}$.

The low response rate might also have limited the validity of this study. The study population was slightly over-representative of clerical and married workers in the target population. However, there were no differences in independent and dependent indices (job stressors and CHD risk factor levels) as well as other demographic/ behavioral variables between the analyzed and excluded workers. Thus, it was unlikely that the selection had a large effect on the results.

Third, blood pressure data were obtained by casual blood pressure measurements. Casual blood pressure measurements are less sensitive than ambulatory blood pressure monitoring in determining an association between psychosocial job characteristics and blood pressure $^{42-44)}$. In addition, serum samples were not collected after fasting in the present study, which might have resulted in measurement errors for serum lipids. Therefore, it is possible that the associations among psychosocial job stressors and CHD risk factors were underestimated in this study.

Fourth, not much consideration was given in this study to the following confounding factors. In this study sample, most workers carried domestic burdens as housekeepers, child care providers, or elderly care providers. Such domestic burdens might have affected the physiological data and shown a relationship with job stress. This study did not measure physical activity at work to adjust for other factors, which limits our interpretation of the unexpected associations of extrinsic effort and job strain with CHD risk factors. It is clear that there are other important possibly confounding factors, which were not included in the present analysis, such as family income, menstruation, dietary pattern, and sedentary lifestyle.

Finally, problems might have resulted due to multiple testing. In this study, the associations between multiple indicators were tested: four to six at once with regards to job stressors and six CHD risk factors. This possibly would increase the $\alpha$ error. Some subtle associations might have been observed as statistically significant.

Our findings did not fully support the hypothesis that an adverse psychosocial work environment is associated with unfavorable CHD risk factors. However, this does 
not mean that the association is not worthy of further study. Rather, our trial should be replicated using a larger employee sample engaged in contingent work characterized by low-wages and poor work prospects and performed mainly by women.

\section{References}

1) RA Karasek: Job demands, job decision latitude, and mental strain: implications for job redesign. Adm Sci Quart 24, 285-308 (1979)

2) H Kuper and M Marmot: Job strain, job demands, decision latitude, and risk of coronary heart disease within the Whitehall II study. J Epidemiol Community Health 57, 147-153 (2003)

3) PL Schnall, PA Landsbergis and D Baker: Job strain and cardiovascular disease. Ann Rev Public Health 15, 381-411 (1994)

4) JV Johnson and EM Hall: Job strain, work place social support, and cardiovascular disease: A cross-sectional study of a random sample of the Swedish working population. Am J Public Health 78, 1336-1342 (1988)

5) N Kawakami, T Haratani and S Araki: Job strain and arterial blood pressure, serum cholesterol, and tobacco use as risk factors for coronary heart disease in Japan. Int Arch Occup Environ Health 71, 429-432 (1998)

6) A Tsutsumi, K Kayaba, K Tsutsumi and M Igarashi: Association between job strain and prevalence of hypertension: a cross-sectional analysis in a Japanese working population with a wide range of occupations: the Jichi Medical School cohort study. Occup Environ Med 58, 367-373 (2001)

7) G Cesana, R Sega, M Ferrario, P Chiodini, G Corrao and G Mancia: Job strain and blood pressure in employed men and women: a pooled analysis of four northern Italian population samples. Psychosom Med 65, 558-563 (2003)

8) CT Su, HJ Yang, CF Lin, MC Tsai, YH Shieh and WT Chiu: Arterial blood pressure and blood lipids as cardiovascular risk factors and occupational stress in Taiwan. Int J Cardiology 81, 181-187 (2001)

9) I Niedhammer, M Goldberg, A Leclerc, S David, I Bugel and MF Landre: Psychosocial work environment and cardiovascular risk factors in an occupational cohort in France. J Epidemiol Community Health 52, 93-100 (1998)

10) C Pieper, AZ LaCroix and RA Karasek: The relation of psychosocial dimensions of work with coronary heart disease risk factors: a meta-analysis of five United States data bases. Am J Epidemiol 129, 483-494 (1989)

11) L Alfredsson, N Hammar, E Fransson, U Faire, J Hallqvist, A Knutsson, T Nilsson, T Theorell and P Westerholm: Job strain and major risk factors for coronary heart disease among employed males and females in a Swedish study on work, lipids, and fibrinogen. Scand J Work Environ Health 28, 238-248 (2002)

12) A Tsutsumi, K Kayaba, S Ishikawa, T Gotoh, N Nago, S Yamada, M Mizooka, K Sakai, S Hayasaka and the Jichi Medical School Cohort Study Group: Job characteristics and serum lipid profile in Japanese rural workers: The Jichi Medical School Cohort Study. J Epidemiol 13, 63-71 (2003)

13) CT Su: Association between job strain status and cardiovascular risk in a population of Taiwanese whitecollar workers. Jpn Circ J 65, 509-513 (2001)

14) DM Reed, AZ LaCroix, RA Karasek, D Miller and A MacLean: Occupational strain and the incidence of coronary heart disease. Am J Epidemiol 129, 495-502 (1989)

15) B Netterstrom, TS Kristensen, MT Damsgaard, O Olsen and A Sjol: Job strain and cardiovascular risk factors: a cross-sectional study of employed Danish men and women. Br J Ind Med 48, 684-689 (1991)

16) $M$ Ishizaki, $P$ Martikainen, $H$ Nakagawa, $M$ Marmot and The Japan Work Stress and Health Cohort Study Group: Socioeconomic status, workplace characteristics, and plasma fibrinogen level of Japanese male employees. Scand J Work Environ Health 27, 287-291 (2001)

17) H Riese, LJ Van Doornen, IL Houtman and EJ De Geus: Job strain and risk indicators for cardiovascular disease in young female nurses. Health Psychol 19, 429-440 (2000)

18) WA Karlin, E Brondolo and J Schwartz: Workplace social support and ambulatory cardiovascular activity in New York city traffic agents. Psychosom Med 65, 167-176 (2003)

19) PA Landsbergis, PL Schnall, K Warren, TG Pickering and JE Schwartz: Association between ambulatory blood pressure and alternative formulations of job strain. Scand J Work Environ Health 20, 349-363 (1994)

20) D Jonsson, A Rosengren, A Dotevall, G Lappas and L Wihelmsen: Job control, job demands, and social support at work in relation to cardiovascular risk factors in MONICA 1995, Goteborg. J Cardiovasc Risk 6, 379_ 385 (1999)

21) J Siegrist: Adverse health effects of high effort/low reward conditions. J Occup Health Psychol 1, 27-41 (1996)

22) H Kuper, A Singh-Manoux, J Siegrist and M Marmot: When reciprocity fails: effort-reward imbalance in relation to coronary heart disease and health functioning within the Whitehall II study. Occup Environ Med 59, 777-784 (2002)

23) H Bosma, R Peter, J Siegrist and M Marmot: Two alternative job stress models and the risk of coronary heart disease. Am J Public Health 88, 68-74 (1998)

24) M Kivimaki, P Leino-Arjas, R Luukkonen, H Riihimaki, J Vahtera and J Kirjonen: Work stress and risk of cardiovascular mortality: prospective cohort study of industrial employees. BMJ 325, 857-860 (2002)

25) R Peter, LAlfredsson, N Hammar, J Siegrist, T Theorell and P Westerholm: High effort, low reward, and cardiovascular risk factors in employed Swedish men and women: baseline results from the WOLF Study. J Epidemiol Community Health 52, 540-547 (1998)

26) Japan Ministry of Public Management, Home Affairs, 
Posts, and Telecommunications. Labor force survey. 2002.

27) B Wilkes, L Stammerjohn and N Lalich: Job demands and worker health in machine-paced poultry inspection. Scand J Work Environ Health 7 Suppl 4, 12-19 (1981)

28) Y Noborisaka, M Ishizaki and Y Yamada: The relationship between job status, gender and workrelated stress amongst middle-aged employees in a computer manufacturing company. J Occup Health 37, 167-168 (1995)

29) I Nyklicek, JJ Vingerhoets and GL Van Heck: Hypertension and objective and self-reported stressor exposure: a review. J Psychosom Res 40, 585-601 (1996)

30) Japanese Society of Hypertension. Japanese society of hypertension guidelines for the management of hypertension. 2000.

31) Japan Atherosclerosis Society. Japan Atherosclerosis Society (JAS) guidelines for diagnosis and treatment of atherosclerotic cardiovascular diseases. 2002.

32) Karasek RA: Job Content Questionnaire and User's Guide. University of Massachusetts at Lowell, Lowell. 1985.

33) N Kawakami, F Kobayashi, S Araki, T Haratani and H Furui: Assessment of job stress dimensions based on the job demands-control model of employees of telecommunication and electric power companies in Japan: reliability and validity of the Japanese version of the job content questionnaire. Int J Behav Med 2, 358-375 (1995)

34) A Tsutsumi, T Ishitake, R Peter, J Siegrist and T Matoba: The Japanese version of the effort-reward imbalance questionnaire: a study in dental technicians. Work and Stress 15, 86-96 (2001)

35) J Siegrist, D Starke, T Chandola, I Godin, M Marmot, I Niedhammer and R Peter: The measurement of effortreward imbalance at work: European comparisons. Soc Sci Med 58, 1483-1499 (2004)

36) RH Rahe, RT Rubin and RJ Arthur: The three investigators study. Serum uric acid, cholesterol, and cortisol variability during stresses of everyday life. Psychosom Med 36, 258-268 (1974)

37) BS McCann, GR Warnick and RH Knopp: Changes in plasma lipids and dietary intake accompanying shifts in perceived workload and stress. Psychosom Med 52, 97-108 (1990)

38) I Mattiasson, F Lindgarde, JA Nilsson and T Theorell: Threat of unemployment and cardiovascular risk factors: longitudinal study of quality of sleep and serum cholesterol concentrations in men threatened with redundancy. BMJ 301, 461-466 (1990)

39) J Siegrist, H Matschinger, P Cremer and D Seidel: Atherogenic risk in men suffering from occupational stress. Atherosclerosis 69, 211-218 (1988)

40) JE Ferrie, MJ Shipley, SA Stansfeld and MG Marmot: Effects of chronic job insecurity and change in job security on self-reported health, minor psychiatric morbidity, physiological measures, and health related behaviors in British civil servants: the Whitehall II study. J Epidemiol Community Health 56, 450-454 (2002)

41) K Belkic, PL Schnall, P Landsbergis and D Baker: The workplace and cardiovascular health: conclusions and thoughts for a future agenda. Occup Med 15, 307-321 (2000)

42) PL Schnall, JE Schwartz, PA Landsbergis, K Warren and TG Pickering: Relation between job strain, alcohol, and ambulatory blood pressure. Hypertension 19, 488494 (1992)

43) T Theorell, G Ahlberg-Hulten, M Jodko, F Sigala and B de la Torre: Influence of job strain and emotion on blood pressure in female hospital personnel during work hours. Scand J Work Environ Health 19, 313318 (1993)

44) LF Van Egeren: The relationship between job strain and blood pressure at work, at home, and during sleep. Psychosom Med 54, 337-343 (1992) 